\title{
Erratum to: Oculo-auriculo-vertebral spectrum: going beyond the first and second pharyngeal arch involvement
}

\author{
Davide Brotto $^{1}$ - Renzo Manara $^{2} \cdot$ Sara Ghiselli $^{1} \cdot$ Elisa Lovo $^{1} \cdot$ Rodica Mardari $^{3}$. \\ Irene Toldo $^{4}$ - Alessandro Castiglione ${ }^{1}$. Giovanni Schifano ${ }^{1}$. Valentina Stritoni ${ }^{5}$. \\ Roberto Bovo ${ }^{1} \cdot$ Patrizia Trevisi $^{1} \cdot$ Alessandro Martini $^{1}$
}

Published online: 31 March 2017

(C) Springer-Verlag Berlin Heidelberg 2017

\section{Erratum to: Neuroradiology}

DOI 10.1007/s00234-017-1795-1

In the article "Oculo-auriculo-vertebral spectrum: going beyond the first and second pharyngeal arch involvement", the list of author names was incorrect. Author names were inverted with the surname preceding first name.

The correct author list is as follows:

Davide Brotto $^{1}$, Renzo Manara ${ }^{2}$, Sara Ghiselli ${ }^{1}$, Elisa Lovo ${ }^{1}$, Rodica Mardari ${ }^{3}$, Irene Toldo ${ }^{4}$, Alessandro Castiglione ${ }^{1}$, Giovanni Schifano ${ }^{1}$, Valentina Stritoni ${ }^{5}$, Roberto Bovo ${ }^{1}$, Patrizia Trevisi ${ }^{1}$, Alessandro Martini ${ }^{1}$

The online version of the original article can be found at http://dx.doi. org/10.1007/s00234-017-1795-1

Davide Brotto

davidebrotto@hotmail.it

1 Department of Neurosciences, Otorhinolaryngology Unit, University of Padova, Via Giustiniani 2, 35128 Padova, Italy

2 Neuroradiology, Sezione di Neuroscienze, University of Salerno, Salerno, Italy

3 Neuroradiology, University Hospital of Padova, Padova, Italy

4 Pediatric Neurology Unit, Department of Woman's and Child's Health, University of Padova, Padova, Italy

5 TIPED, Department of Pediatrics, University of Padova, Padova, Italy 